\title{
Complications and local relapse after intraoperative low-voltage X-ray radiotherapy in breast cancer
}

\author{
Ana Alicia Tejera Hernández ${ }^{1,2}$, Víctor Manuel Vega Benítez ${ }^{1,2}$, Juan Carlos Rocca Cardenas ${ }^{1}$, Neith Ortega Pérez ${ }^{1,2}$, \\ Nieves Rodriguez Ibarria ${ }^{1,3}$, Juan Carlos Díaz Chico', Juan José García-Granados Alayón ${ }^{1,4}$, Pedro Pérez Correa ${ }^{1,2}$, \\ Juan Ramón Hernández Hernández ${ }^{1,2}$
}

${ }^{1}$ Faculty of Health Sciences, University of Las Palmas de Gran Canaria, Las Palmas, Spain

${ }^{2}$ General Surgery Department, Hospital Universitario Insular de Gran Canaria, Las Palmas de Gran Canaria, Las Palmas, Spain

${ }^{3}$ Therapeutic Radiation and Oncology Department, Hospital Universitario de Gran Canaria Dr Negrin, Las Palmas, Spain

${ }^{4}$ Service of Radiophysics and Radiological Protection, Hospital Universitario Insular de Gran Canaria, Las Palmas, Spain

\begin{abstract}
Purpose: To study those factors that influence the occurrence of surgical complications and local relapse in patients intervened for breast cancer and receiving intraoperative radiotherapy.

Methods: Observational study on patients intervened for breast cancer with conservative surgery and intraoperative radiotherapy with low-voltage X-ray energy source (INTRABEAM), from 2015 to 2017 with 24 months minimum followup. Variables possibly associated to the occurrence of postoperative complications were analyzed with the Student t-test and the Fisher exact test; $P<0.05$ considered significant. Subsequently, the construction of multiple multivariate analysis models began, thus building a logistic regression analysis using the IBM SPSS Statistics ver. 23 software. Local relapse was described.

Results: The study included 102 patients, mean age of 61.2 years; mean global size of tumor, 12.2 mm. Complications occurred in $29.4 \%$. Fibrosis was the most frequently observed complication, followed by postoperative seroma. Using a 45 $\mathrm{mm}$ or larger applicator were significantly associated with the occurrence of complications. Tumor size $2 \mathrm{~cm}$ or larger and reintervention showed borderline significant association. Only one case of local relapse was observed.

Conclusion: Certain factors may increase the risk of complication after the use of intraoperative radiotherapy. Using external complementary radiotherapy does not seem to increase the rate of complications. Select patients and the involvement of a multidisciplinary team are essential for achieving good results.

[Ann Surg Treat Res 2020;98(6):299-306]
\end{abstract}

Key Words: Breast neoplasms, Complications, Local neoplasm recurrence, Radiotherapy

\section{INTRODUCTION}

Intraoperative radiotherapy (IORT) is based on the administration, during surgical intervention, of a single dose of ionizing radiation directly on the surgical tumorectomy cavity, with the aim of enhancing local control of the disease, while reducing secondary toxicity to the surrounding tissues, which results from radiation [1]. This technique enhances identification of an exact location to apply the radiation boost and reduces the time interval between surgery and radiation. Given that patients are radiated while they are under anesthesia, errors occasioned by the patient's movements or by wrong positioning
Received October 28, 2019, Revised March 8, 2020,

Accepted April 7, 2020

Corresponding Author: Ana Alicia Tejera Hernández

General Surgery Department, Hospital Universitario Insular de Gran Canaria, Avda. Marítima del Sur, 35016 Las Palmas de Gran Canaria, Las Palmas, Spain

Tel: +34-928-44-1652, Fax: +34-928-44-1651

E-mail: anath15@hotmail.com

ORCID: https://orcid.org/0000-0002-5796-2732

\section{Copyright (c) 2020, the Korean Surgical Society}

(c) Annals of Surgical Treatment and Research is an Open Access Journal. All articles are distributed under the terms of the Creative Commons Attribution NonCommercial License (http://creativecommons.org/licenses/by-nc/4.0/) which permits unrestricted non-commercial use, distribution, and reproduction in any medium, provided the original work is properly cited. 
are almost inexistent [2]. This technique has been used both as a boost to conventional external radiotherapy (ERT) and as the only treatment at initial stages of breast cancer [2,3]. The biological efficacy of an IORT dose is equivalent to that of the conventional administration of an ERT dose fractionated 2-3 times higher. When a 45- to 50-Gy doses of ERT is used in combination with a 10- to 20-Gy dose of IORT, higher rates of local control of the disease are attained, especially regarding the control of residual disease [4].

There are different types of IORT. Using a 50-kV X-ray energy source is a standard procedure carried out in a number of hospitals in our area, whose short- and long-term surgical complications have been scarcely described in the current literature [5,6]. Possible complications from a conservative surgical intervention for breast cancer might worsen from using this type of radiation [7]. Thus, studying demographic and technical factors that may influence such complications is essential for adequate planning of a multidisciplinary treatment.

The goal of this study was to establish what factors influence the occurrence of surgical complications in patients undergoing breast cancer surgical intervention, associated with low-voltage X-ray IORT. Additionally, identification of possible cases of local relapse during follow-up was pursued.

\section{METHODS}

This was an observational study with consecutive sampling, on patients intervened for breast cancer with conservative surgery and IORT with low-voltage X-ray energy source (máximo $50 \mathrm{kV}$ ) con el dispositivo Intrabeam (Carl Zeiss Meditec, Oberkochen, Alemania), from 2015 to 2017 with 24 months minimum follow-up, which terminated in 2019. A group of irradiated patients, who presented postoperative complications (IORT-COM) were compared with a second group of patients, who had been irradiated with the same technique but did not present complications in follow-up (IORT). Furthermore, the number of patients presenting local relapse and the characteristics possibly influencing its occurrence were determined.

Inclusion criteria were selected following the protocol of the multicenter controlled randomized clinical trial TARGIT-A. This study presents a long-term follow-up and its criteria are the most used internationally in the application of intraoperative low-voltage X-ray radiotherapy [2,3]. Based on this, the inclusion criteria were: patients had to be 46 years old or older, with unifocal tumor of $3 \mathrm{~cm}$ or smaller, histological results corresponding to infiltrating carcinoma (except lobular), clinically and radiologically negative results in axilla and positive hormonal receptors.

All patients were managed with the same irradiation technique. An initial measurement of the cavity was carried out with a simulation $\mathrm{CT}$ in order to estimate the magnitude of the volume to be irradiated and the applicator to be used. Subsequently, tumorectomy was conducted and the breast tissue around the cavity was adjusted to the sphere used to apply radiation by making a tobacco-pouch. After verifying that the placement was correct and the margins were adequate, through intraoperative assessment, a single radiation dose of 20 Gy was applied on the applicator surface, which corresponded to 5-7 Gy at 1-cm depth. Dosage and treatment time varied depending on the applicator size. Finally, the applicator was removed and the incision was closed. The treatments lasted from 15 to 45 minutes depending on the source and the applicator.

Subsequently, depending on the definitive histological findings (unexpected lobular carcinoma, surgical margins involved, lymph node involvement, or surgical reintervention), additional complementary ERT could be applied. Patients, who failed to complete the follow-up, those who did not meet the inclusion criteria, and those who refused to complete the complementary treatments established in the above protocol, were excluded from the study. Patients were followed up in outpatient clinics and their data were collected retrospectively from the institutional database.

Complication was defined for any alteration of the expected course of both the local and systemic response [8]. The identification of this was done by the surgeon through direct observation during the entire postoperative follow-up of the patient (2 years).

Complications which emerged during the follow-up period were identified (seroma, hematoma, infection, dehiscence, necrosis, or fibrosis) and described as percentages according to their relevance. Additionally, the relationship between the occurrence of complications and the use of complementary ERT was studied, in order to evaluate possible differences between patients managed exclusively with intraoperative radiotherapy (IORT-EX) and those requiring complementary external radiotherapy (IORT-CER).

Demographic variables were classified and studied dichotomously: age ( $\geq 70$ years), size ( $\geq \mathrm{T} 2)$, axillary

Table 1. Mean age, applicator size and months freer of disease $(\mathrm{n}=102)$

\begin{tabular}{lcrl}
\hline \multicolumn{1}{c}{ Variable } & $\begin{array}{c}\text { IORT } \\
(\mathrm{n}=72)\end{array}$ & $\begin{array}{c}\text { IORT-COM } \\
(\mathrm{n}=30)\end{array}$ & P-value \\
\hline Age $(\mathrm{yr})$ & $60.86 \pm 8.05$ & $61.6 \pm 8.72$ & 0.91 \\
Applicator size $(\mathrm{mm})$ & $38.06 \pm 5.47$ & $38.67 \pm 5.24$ & 0.43 \\
Period free of disease $(\mathrm{mo})$ & $28.88 \pm 9.83$ & $29.6 \pm 10.6$ & 0.46 \\
\hline
\end{tabular}

Values are presented as mean \pm standard deviation.

IORT, intraoperative radiotherapy; COM, complications. 
Table 2. Patient characteristics

\begin{tabular}{|c|c|c|c|}
\hline \multirow{2}{*}{ Variable } & \multicolumn{2}{|c|}{ Complications } & \multirow{2}{*}{ P-value } \\
\hline & Absent (IORT) & Present (IORT-COM) & \\
\hline No. of cases & $72(71)$ & $30(29)$ & \\
\hline Age (yr) & & & 0.860 \\
\hline$<70$ & $61(85)$ & $25(83)$ & \\
\hline$\geq 70$ & $11(15)$ & $5(17)$ & \\
\hline Tumor size $(\mathrm{mm})$ & & & 0.053 \\
\hline$<20$ & $67(93)$ & $24(80)$ & \\
\hline$\geq 20$ & $5(7)$ & $6(20)$ & \\
\hline Lymph node status & & & 0.494 \\
\hline Positive & $19(26)$ & $6(20)$ & \\
\hline Negative & $53(74)$ & $24(80)$ & \\
\hline Radioguided surgery & & & 0.119 \\
\hline Yes & $31(43)$ & $18(60)$ & \\
\hline No & $41(57)$ & $12(40)$ & \\
\hline Applicator size (mm) & & & 0.028 \\
\hline$<45$ & $60(83)$ & $19(63)$ & \\
\hline$\geq 45$ & $12(17)$ & $11(37)$ & \\
\hline Ductal carcinoma in situ & & & 0.412 \\
\hline Yes & $32(44)$ & $16(53)$ & \\
\hline No & $40(56)$ & $14(47)$ & \\
\hline Histological grade & & & 0.366 \\
\hline$<\mathrm{G} 2$ & $43(60)$ & $15(50)$ & \\
\hline$\geq \mathrm{G} 2$ & $29(40)$ & $15(50)$ & \\
\hline Lymphovascular involvement & & & 0.192 \\
\hline Yes & $26(36)$ & $15(50)$ & \\
\hline No & $46(64)$ & $15(50)$ & \\
\hline Progesterone receptor & & & 0.592 \\
\hline Positive & $62(86)$ & $27(90)$ & \\
\hline Negative & $10(14)$ & $3(10)$ & \\
\hline Ki67\% & & & 0.178 \\
\hline Positive & $24(33)$ & $6(20)$ & \\
\hline Negative & $48(66)$ & $24(80)$ & \\
\hline HER2 & & & 0.431 \\
\hline Positive & $7(10)$ & $1(3)$ & \\
\hline Negative & $65(90)$ & $29(97)$ & \\
\hline Margins (mm) & & & 0.500 \\
\hline$<1$ & $17(24)$ & $9(30)$ & \\
\hline$\geq 1$ & $55(76)$ & $21(70)$ & \\
\hline Reintervention & & & 0.075 \\
\hline Yes & $1(1)$ & $3(10)$ & \\
\hline No & $71(99)$ & $27(90)$ & \\
\hline Adjuvant chemotherapy & & & 0.259 \\
\hline Yes & $25(35)$ & $7(23)$ & \\
\hline No & $47(65)$ & $23(77)$ & \\
\hline Complementary external radiotherapy & & & 0.776 \\
\hline Yes & $31(43)$ & $12(40)$ & \\
\hline No & $41(57)$ & $18(60)$ & \\
\hline Local relapse & & & 0.517 \\
\hline Yes & $1(1)$ & $0(0)$ & \\
\hline No & $71(99)$ & $30(100)$ & \\
\hline
\end{tabular}

Values are presented as number (\%).

IORT, intraoperative radiotherapy; COM, complications. 
involvement, presence of associated in situ carcinoma, lymphovascular infiltration (LVI), histological grade, ki $67 \%$, hormonal receptors, Her 2, close margin $(\leq 1 \mathrm{~mm})$, radioguided surgery, applicator size ( $\geq 45 \mathrm{~mm})$; as well as the occurrence of complications, surgical reintervention, use of complementary ERT, chemotherapy or local relapse.

Variables possibly associated with the occurrence of postoperative complications were analyzed by using the Student t-test for numerical variables and the Fisher exact test for dichotomous ones; $\mathrm{P}<0.05$ was considered significant. Subsequently, the construction of multiple multivariate analysis models began, thus building a logistic regression analysis. The statistical analysis was conducted with the IBM SPSS Statistics ver. 23.0 (IBM Co., Armonk, NY, USA). Finally, the main characteristics of patients with relapse were evaluated, and possible differences, as compared to patients without relapse, were identified.

This study was approved by Complejo Hospitalario Universitario Insular Materno Infantil Institution Review Board (IRB) with the number 2019-293-1.

\section{RESULTS}

The study included 102 patients: 72 IORT and 30 IORTCom (Table 1). Both groups showed similar demographic characteristics with a mean age of 61.2 years, without significant differences. The mean follow-up time was 29.2 months, similar for both groups (p:NS). The mean global size of tumors was $12.2 \mathrm{~mm}$ and the most frequently used applicator size was 40 $\mathrm{mm}$ (34 patients) closely followed by $35 \mathrm{~mm}$ (33 patients). The reintervention rate was $3.92 \%$ and all reinterventions were needed due to involvement of the surgical margins.

A proportion of $29.4 \%$ of patients presented complications (Table 2): seroma, hematoma, infection, dehiscence, necrosis, or fibrosis. Such complications did not require surgical intervention. The use of an applicator of $45 \mathrm{~mm}$ or larger was an independent factor significantly associated to the occurrence of surgical complications $(P \leq 0.05)$. Tumor size of $2 \mathrm{~cm}$ or larger $(\mathrm{P}=0.053)$ and the need for reintervention $(\mathrm{P}=0.075)$ showed borderline significant association. The remaining variables were not significantly associated. The logistic regression analysis (Table 3) revealed that only the use of an applicator of $45 \mathrm{~mm}$ or larger was a statistically significant variable, and the most relevant one obtained in the study.

Table 4 describes the complication types. They occurred independently or in combination. Results are expressed individually. Fibrosis was the most frequent complication (17\%) and it appeared generally late in these patients (4 to 6 months postoperative); seroma was the second most frequent complication and occurred in $11 \%$ of cases; infection, hematoma, dehiscence, and necrosis were rare complications. No significant differences were observed between the complication rates

Table 3. Logistic regression analysis

\begin{tabular}{lcccc}
\hline \multicolumn{1}{c}{ Variable $^{\mathrm{a})}$} & Wald & Exp (B) & $95 \% \mathrm{Cl}$ & P-value \\
\hline Tumor size $\geq 20 \mathrm{~mm}$ & 3.076 & 0.297 & $0.077-1.153$ & 0.079 \\
Applicator size $\geq 45 \mathrm{~mm}$ & 4.784 & 0.323 & $0.118-0.889$ & 0.029 \\
Reintervention & 3.098 & 0.114 & $0.010-1.279$ & 0.078 \\
\hline
\end{tabular}

$\mathrm{Cl}$, confidence interval.

${ }^{a}$ Statistically significant variables in the univarible analysis.

Table 4. Types of complication ${ }^{\text {a) }}$

\begin{tabular}{lcrr}
\hline \multicolumn{1}{c}{ Variable } & \multicolumn{2}{c}{ Complementary external radiotherapy } & P-value \\
& Absent (IORT-EX) & Present (IORT-CER) & \\
No. of cases & $59(58)$ & $43(42)$ & 0.530 \\
Fibrosis $(17 \%)$ & $11(19)$ & $4(9)$ & 0.757 \\
Seroma $(11 \%)$ & $7(12)$ & $2(5)$ & $>0.999$ \\
Infection $(5.8 \%)$ & $4(7)$ & $1(2)$ & $>0.999$ \\
Hematoma $(2.9 \%)$ & $2(3)$ & $1(2)$ & $>0.999$ \\
Dehiscence $(2.9 \%)$ & $2(3)$ & $0(0)$ & $>0.999$ \\
Necrosis $(0.9 \%)$ & $1(2)$ & & \\
\hline
\end{tabular}

Values are presented as number (\%).

IORT, intraoperative radiotherapy; EX, exclusive; CER, complementary external radiotherapy.

${ }^{\text {a) }}$ Some patients may have more than one complication at a time. 
with or without complementary ERT. Only one case of local relapse was observed in a patient with a 42-month period free of disease, without significant differences as compared with the rest of the patients. No cases of distant disease were recorded.

\section{DISCUSSION}

Irradiating the surgical bed during the surgical procedure is a rapid, attractive option that allows for direct localization of the tumor bed and the application of both treatments on the same day. Radiation is accurately applied on the area with the highest risk of tumor relapse, while preserving the healthy surrounding tissue [2,3]. Some $15 \%-30 \%$ of patients are unable to complete an adjuvant treatment with ERT due to their advanced age, associated comorbidities, or distance from home to the reference hospital; all of which increases their probability of suffering local relapse $[9,10]$. Such patients may directly benefit from this technique. The patient-tailored dosage in this technique reduces the risk of complications and toxicity. However, higher rates of certain, characteristic complications have been described [7,10]. In our study, both patient groups were homogeneous regarding mean follow-up time, mean age, and months free of disease; thus, our sample was homogeneous and both groups were comparable.

Elderly patients are at higher risk of developing complications due to associated comorbidities, complementary medication or age-related physiological changes, which might impair immune response and wound healing [11]. This variable was not significant in the study. Tumors larger than $2 \mathrm{~cm}$ require removal of a considerable amount of breast tissue [12,13]. In such cases, the size of the remaining cavity requires a larger applicator; furthermore, longer application time is needed to reach an optimal dose, which may in turn prolong the operation time [14]. All of these characteristics contributed to the statistical significance of this factor in our sample.

Radioguided surgery for nonpalpable tumors may be conducted with hookwires or through radioactive isotopes. Since they are small, easy-to-locate lesions, small resections can be performed; thus reducing postoperative complications $[2,13]$. The need for axillary emptying or for application of complementary ERT may increase such complications $[15,16]$. Both of these factors were not statistically significant.

The applicator is chosen on the basis of the preoperative simulation CT and intraoperative measurements of the surgical bed. The larger the cavity, the harder is it to choose a suitable applicator. Larger applicator size has been associated to higher complication rates [17]. It is associated with more severe surgical injury, increased risk of bleeding, and larger irradiation area with possible higher risk of subsequent fibrosis [17,18]. This was the most relevant factor in our study.

The presence of associated in situ carcinoma is related to the need for more reinterventions to achieve free margins and possibly to larger extension of the disease [19]. In our study, it was not a significant factor. Immunohistochemical factors such as LVI, histological grade, ki 67\%, hormonal receptors, Her 2, were studied as an integrated part of the study, in order to assess their relationship with possible subsequent relapse, and were not significantly related to the occurrence of complications. Close, but not involved, margins may require higher radiation administered through complementary ERT [2,3]. Such complementary radiation is related to higher toxicity and increased local heat and redness, with subsequent fibrosis and scar retraction [20]. Margins smaller than $1 \mathrm{~mm}$ or the use of complementary ERT because of these margins were not significant factors in our study.

The need for surgical reintervention increases the risk of complications. Capillary regeneration and neovascularization are interrupted and the possibility of bleeding is higher. Although the previous scar can be used to conduct the surgical reintervention, the act of removing more breast tissue will affect the final esthetic outcome [7,11]. If the wound edges are not freshened, more necrosis of the skin flaps may occur with dehiscence of the surgical wound. Reopening the surgical cavity increases the degree of contamination and the infection rate $[7,10,21]$. Furthermore, a second surgical intervention produces a physical and psychological impact on patients, doubtlessly affecting their quality of life. This variable, surgical reintervention, showed a borderline statistical significance in our study. Enlarging our sample to a larger amount of cases would result in a statistical tendency towards definitive significance. We consider it a relevant factor in preventing the occurrence of complications. Adjuvant chemotherapy is used in patients with a higher cell proliferation index, axillary involvement or unfavorable biological factors, and it does not seem to have an influence on the occurrence of complications.

There are several types of surgical complications. The variability of complication rates in published studies stems from the criteria used to consider an adverse event to be a complication, and range from $5 \%$ to $40 \%$ of cases $[6,10,15,22]$. Our rate was similar to those reported in the most current literature. We included all complications requiring medical treatment, aspiration, draining or debridement (Clavien Dindo I-II) [22]. No severe complications occurred. No significant rate of difference was found between patients with or without subsequent ERT, for any complication.

Fibrosis, or late-onset induration, was the most frequent complication. It is related to chronic nonsevere toxicity, which occurs in most patients receiving an IORT boost plus subsequent complementary ERT $[23,24]$. In our series, no significant differences were found in the occurrence of such complications, depending on the type of schedule used. Since this is a late-onset complication, controversy exists on whether 
it should be considered a postoperative complication or a long-term consequence of radiotherapy, which might worsen with the years and become associated with retraction of the intervened zone and reduction of the global size of the breast.

IORT increased the occurrence of postsurgical seroma [15,25]. Early closing of the surgical cavity by breast remodeling, which we systematically carry out, could account for the lower amount of seromas observed in our series, as compared with other published series. In the beginnings of this technique, there was a tendency to remove a larger amount of healthy tissue, in order to guarantee free margins, which increased seromas. With more experience, it became clear that it was not necessary to modify the tumorectomy technique because of the use of IORT. This type of seroma usually appears late, and less than $5 \%$ of cases require more than 2 aspirations for resolution [6,15,25].

The time the cavity remains open for performing the technique increases the risk of subsequent contamination of the surgical wound $[18,26]$. Although our results are adequate, we currently use antibiotic prophylaxis in such cases, even when the procedure is considered clean surgery. During the technique, a tobacco-pouch is necessary to adjust the cavity to the applicator, which means more surgical manipulation of the tissue and may increase hematoma. In our series, the amount of hematomas requiring treatment was low. Cases presenting dehiscence or necrosis of the skin flaps occurred in patients with wound infection managed with drainage.

Since only one case of local relapse was observed, statistically relevant prognostic factors are difficult to obtain. The involved patient was younger than 70 years at the time of diagnosis, size was smaller than T2, without axillary involvement, with associated in situ carcinoma, LVI, high histological grade, ki $67 \%$ positive, progesterone receptor negative, Her 2 negative, free margins, applicator size $35 \mathrm{~mm}$, without complications or need for reintervention. During follow-up, chemotherapy and hormonal therapy were used as adjuvant treatment. Some of the above-mentioned factors (associated in situ carcinoma, LVI, high histological grade, high ki 67\% and progesterone receptor negative) are typical factors known to be related to local relapse [27-30].

In general, the results of our study should be considered cautiously, since they correspond to the experience in only one center and with a limited number of cases. Although the followup time was long, an even longer period might have yielded more cases of local relapse. The assessment and classification of complications is made by surgeons and, although based on objective criteria, some degree of subjectivity, variability in time and surgeon's experience, may exist. The here-presented results include the first cases managed in our center; thus, the learning curve might have prolonged the surgical times, possibly resulting in more complications.

Based on these results, we consider that IORT is a safe and effective technique, which can be easily reproduced and should be used in most breast-units where the necessary equipment is available. Complications are similar to those of a traditional conservative treatment. A considerable proportion of patients will need to complete the treatment with ERT, although it does not seem to increase complications. Regarding local relapse, long-term follow-up is needed to determine whether the technique actually increases its rate; however, up to this date, available patient series do not show such a phenomenon.

In conclusion, fibrosis, or late-onset induration, is the most frequently observed complication, followed by postoperative seroma. The use of an applicator of $45 \mathrm{~mm}$ or larger was an independent factor significantly associated to the occurrence of surgical complications. Tumor size of $2 \mathrm{~cm}$ or larger and the need for reintervention showed borderline significant association. These factors must be taken into account to prevent their occurrence and to apply an early treatment to reduce psychological impact and enhance patient quality of life. The use of complementary ERT does not seem to increase the complication rate. IORT does not increase the risk of local relapse in the medium-term, although larger patient series and longer follow-up periods are needed to demonstrate this. Patient selection and the involvement of a multidisciplinary team are essential for achieving good results.

\section{ACKNOWLEDGEMENTS}

\section{Conflict of Interest}

No potential conflict of interest relevant to this article was reported.

\section{ORCID iD}

Ana Alicia Tejera Hernández:

https://orcid.org/0000-0002-5796-2732

Víctor Manuel Vega Benítez:

https://orcid.org/0000-0002-0596-3880

Juan Carlos Rocca Cardenas:

https://orcid.org/0000-0003-3100-6707

Neith Ortega Pérez: https://orcid.org/0000-0002-9803-1328

Nieves Rodriguez Ibarria:

https://orcid.org/0000-0003-1564-2703

Juan Carlos Díaz Chico:

https://orcid.org/0000-0002-0944-990X

Juan José García-Granados Alayón:

https://orcid.org/0000-0001-8774-9222

Pedro Pérez Correa: https://orcid.org/0000-0001-9686-7503

Juan Ramón Hernández Hernández:

https://orcid.org/0000-0001-8854-8078

Author Contribution

Conceptualization: AATH, VMVB, JRHH 
Formal Analysis: JCDC, JCRC, AATH

Investigation: AATH, VMVB, JCRC, NOP, NRI, JJGGA, PPC,

JRHH

Methodology: AATH, VMVB, JCDC, JRHH
Project Administration: AATH, VMVB, JRHH

Writing - Original Draft: AATH

Writing - Review \& Editing: AATH

\section{REFERENCES}

1. Orecchia R, Ciocca M, Lazzari R, Garibaldi C, Leonardi MC, Luini A, et al. Intraoperative radiation therapy with electrons (ELIOT) in early-stage breast cancer. Breast 2003;12:483-90.

2. Vaidya JS, Joseph DJ, Tobias JS, Bulsara M, Wenz F, Saunders C, et al. Targeted intraoperative radiotherapy versus whole breast radiotherapy for breast cancer (TARGIT-A trial): an international, prospective, randomised, non-inferiority phase 3 trial. Lancet 2010;376:91-102.

3. Vaidya JS, Wenz F, Bulsara M, Tobias JS, Joseph DJ, Keshtgar M, et al. Risk-adapted targeted intraoperative radiotherapy versus whole-breast radiotherapy for breast cancer: 5-year results for local control and overall survival from the TARGIT-A randomised trial. Lancet 2014;383:603-13.

4. Smith BD, Arthur DW, Buchholz TA, Haffty BG, Hahn CA, Hardenbergh PH, et al. Accelerated partial breast irradiation consensus statement from the American Society for Radiation Oncology (ASTRO). J Am Coll Surg 2009;209:269-77.

5. Morlino A, La Torre G, Lapadula L, Cammarota A. IORT in breast cancer. Our experience of the first patients treated. Ann Ital Chir 2017;88:253-7.

6. Rakhra S, Bethke K, Strauss J, Hayes JP, Hansen N, Khan SA, et al. Risk factors leading to complications in early-stage breast cancer following breast-conserving surgery and intraoperative radiotherapy. Ann Surg Oncol 2017;24:1258-61.

7. Gulcelik MA, Dogan L, Karaman N, Turan M, Kahraman YS, Akgul GG, et al. Intraoperative boost radiation effects on early wound complications in breast cancer patients undergoing breast- conserving surgery. Turk J Med Sci 2017;47:1185-90.

8. de Glas NA, Kiderlen M, Bastiaannet E, de Craen AJ, van de Water W, van de Velde CJ, et al. Postoperative complications and survival of elderly breast cancer patients: a FOCUS study analysis. Breast Cancer Res Treat 2013;138:561-9.

9. Athas WF, Adams-Cameron M, Hunt WC, Amir-Fazli A, Key CR. Travel distance to radiation therapy and receipt of radiotherapy following breast-conserving surgery. J Natl Cancer Inst 2000;92:269-71.

10. Epstein M, Silverstein M, Lin K, Kim B, Khan S, De Leon C, et al. Acute and chronic complications in breast cancer patients treated with intraoperative radiation therapy. Ann Surg Oncol 2016;23:3304-9.

11. Tesarova P. Specific aspects of breast cancer therapy of elderly women. Biomed Res Int 2016;2016:1381695.

12. Hwang KT, Han W, Lee SM, Choi J, Kim J, Rhu J, et al. Prognostic influence of 3-dimensional tumor volume on breast cancer compared to conventional 1-dimensional tumor size. Ann Surg Treat Res 2018;95:183-91.

13. Fei F, Messina C, Slaets L, Chakiba C, Cameron D, Bogaerts J, et al. Tumour size is the only predictive factor of distant recurrence after pathological complete response to neoadjuvant chemotherapy in patients with large operable or locally advanced breast cancers: a sub-study of EORTC 10994/BIG 1-00 phase III trial. Eur J Cancer 2015:51:301-9.

14. Sethi A, Emami B, Small W Jr, Thomas TO. Intraoperative radiotherapy with INTRABEAM: technical and dosimetric considerations. Front Oncol 2018;8:74.
15. Ebner F, Schramm A, Bottke D, Friedl TW, Wiegel T, Fink V, et al. Comparison of seroma production in breast conserving surgery with or without intraoperative radiotherapy as tumour bed boost. Arch Gynecol Obstet 2016;294:861-6.

16. Meiers P, Cil T, Guller U, Zuber M. Sentinel lymph node biopsy in early-stage breast cancer patients: improved survival through better staging? Langenbecks Arch Surg 2013;398:687-90.

17. Lee JJB, Choi J, Ahn SG, Jeong J, Lee IJ, Park $\mathrm{K}$, et al. In vivo dosimetry and acute toxicity in breast cancer patients undergoing intraoperative radiotherapy as boost. Radiat Oncol J 2017;35:121-8.

18. Valente SA, Fanning A, Stewart RA, Grundfest S, Tendulkar RD, Cherian $\mathrm{S}$, et al. Intraoperative radiation for breast cancer with Intrabeam ${ }^{\mathrm{TM}}$ : factors associated with decreased operative times in patients having IORT for breast cancer. Front Oncol 2017;7:237.

19. Zhang X, Dai H, Liu B, Song F, Chen K. Predictors for local invasive recurrence of ductal carcinoma in situ of the breast: a meta-analysis. Eur J Cancer Prev 2016;25:19-28.

20. Key S, Miglierini P, Dupre PF, Guilbert S, Lucia AS, Abgral R, et al. Cosmetic outcome and chronic breast toxicity after intraoperative radiation therapy (IORT) as a single modality or as a boost using the Intrabeam(®) device: a prospective study. Ann Surg Oncol 2017:24:2547-55.

21. Zur M, Shai A, Leviov M, Bitterman A, Shiloni E, Ben Yosef R, et al. Shortterm complications of intra-operative radiotherapy for early breast cancer. J Surg Oncol 2016;113:370-3.

22. Panhofer P, Ferenc V, Schutz M, Gleiss A, 
Dubsky P, Jakesz R, et al. Standardization of morbidity assessment in breast cancer surgery using the Clavien Dindo Classification. Int J Surg 2014;12:334-9.

23. Cracco S, Semprini G, Cattin F, Gregoraci G, Zeppieri M, Isola M, et al. Impact of intraoperative radiotherapy on cosmetic outcome and complications after oncoplastic breast surgery. Breast J 2015:21:285-90.

24. Sedlmayer F, Reitsamer R, Wenz F, Sperk E, Fussl C, Kaiser J, et al. Intraoperative radiotherapy (IORT) as boost in breast cancer. Radiat Oncol 2017;12:23.

25. Tuschy B, Berlit S, Romero S, Sperk E, Wenz F, Kehl S, et al. Clinical aspects of intraoperative radiotherapy in early breast cancer: short-term complications after IORT in women treated with low energy x-rays. Radiat Oncol 2013;8:95.

26. Silverstein MJ, Epstein M, Kim B, Lin K, Khan S, Snyder L, et al. Intraoperative radiation therapy (IORT): a series of 1000 tumors. Ann Surg Oncol 2018;25:2987-93.

27. Hasebe T, Iwasaki M, Hojo T, Shibata T, Kinoshita T, Tsuda H. Histological factors for accurately predicting first locoregional recurrence of invasive ductal carcinoma of the breast. Cancer Sci 2013;104:1252-61.

28. Pata G, Guaineri A, Bianchi A, Amoroso V, Pasinetti N, Pasini M. Long-term outcomes of immunohistochemically defined subtypes of breast cancer less than or equal to $2 \mathrm{~cm}$ after breast-conserving surgery. J Surg Res 2019;236:288-99.

29. Choi HJ, Ryu JM, Kim I, Nam SJ, Kim $\mathrm{SW}, \mathrm{Yu}$ J, et al. Nomogram for accurate prediction of breast and axillary pathologic response after neoadjuvant chemotherapy in node positive patients with breast cancer. Ann Surg Treat Res 2019;96:169-76.

30. Abbott AM, Dossett LA, Loftus L, Sun W, Fulp W, Sokol GH, et al. Intraoperative radiotherapy for early breast cancer and age: clinical characteristics and outcomes. Am J Surg 2015;210:624-8. 